\title{
Faunistic Study on the Family Meloidae (Coleoptera) of Ankara Province (Turkey)
}

\section{Ankara ili (Türkiye) Meloidae (Coleoptera) Familyası Üzerine Faunistik Çalışma}

\section{Muhammed Arif Demir ${ }^{1 \oplus}$ and Mahmut Kabalak ${ }^{1,2^{*} \oplus}$}

${ }^{1}$ Department of Biology, Hacettepe University, Beytepe, Ankara, Turkey.

${ }^{2}$ Hacettepe University Biodiversity Research and Application Center, Ankara, Turkey.

\section{ABSTRACT}

The main aim of this study is to determining Meloidae fauna of Ankara. 3301 specimens were collected. 43 species belonging to 2 subfamilies and 15 genera were identified. The genus Mylabris Fabricius, 1775 is the most species-rich genus with 10 species. Cerocoma (s.str.) bernhaueri Pardo Alcaide, 1977, Alosimus luteus (Waltl, 1838), Teratolytta monticola Bologna, 2006, Hycleus polymorphus (Pallas, 1771), H. sexmaculatus (A.G. Olivier, 1811), Mylabris (Micrabris) unicolor Faldermann, 1837, Meloe (Eurymeloe) mediterraneus (G. Muller, 1925) and Stenoria (s.str.) apicalis (Latreille, 1804) species were recorded from Central Anatolian Region of Turkey for the first time. Detailed locality records of M. (Micrabris) unicolor and S. (s.str.) apicalis in Turkey were given with this study for the first time. The most western distributions of Alosimus luteus and Teratolytta monticola were given with this study.

\section{Key Words}

Meloidae, Ankara, Turkey, Faunistic evaluations.

u çalışmanın temel amacı Ankara'nın Meloidae faunasını belirlemektir. 3301 örnek toplanmıştır. 2 altfamilya ve 15 cinse 3 ait toplam 43 tür tespit edilmiştir. Mylabris Fabricius cinsi, 1775, 10 türle tür sayısı açısından en zengin cinstir. Cerocoma (s.str.) bernhaueri Pardo Alcaide, 1977, Alosimus luteus (Waltl, 1838), Teratolytta monticola Bologna, 2006, Hycleus polymorphus (Pallas, 1771), H. sexmaculatus (AG Olivier, 1811), Mylabris (Micrabris) unicolor Faldermann, 1837, Meloe (Eurymeloe) mediterraneus (G. Muller, 1925) ve Stenoria (s.str.) apicalis (Latreille, 1804) türleri ilk defa bu çalışma ile Türkiye'nin İç Anadolu Bölgesi'nden kayıt edilmiştir. M. (Micrabris) unicolor ve S. (s.str.) apicalis'in bu çalışma ile Türkiye için ilk defa detaylı lokalite kaydı verilmiştir. Alosimus luteus ve Teratolytta monticola türlerinin en batı kaydı bu çalışma ile verilmiştir.

\section{Anahtar Kelimeler}

Meloidae, Ankara, Türkiye, Faunistik değerlendirmeler. 


\section{INTRODUCTION}

Thef he family Meloidae (Coleoptera) has about 3000 species belonging to 120 genera in the Palearctic Region [1]. This family, also known as blister beetles, is cosmopolitan (except New Zealand, Antarctica and most Polynesian islands) [2]. Species distribute in the temperate steppe, arid regions, semi-tropics, tropical savannas, or other open habitats $[3,4]$.

In Turkey, many researchers have conducted researches on this family. Bologna [5] reported that the greatest contributions to the knowledge of the Turkish Meloidae fauna were made by some German and Austrian researchers such as Ganglauber, Haag-Rutenberg, von Heyden, Kraatz, Prochàzka and Reitter. Researchers such as Escherich, Marseul, Abeille de Perrin and Frivaldszky have also made significant contributions. In addition, revision of genera covering most Mediterranean species by researchers such as Maran, Kaszab, Muche and Alcaida helped to know the Turkish Meloidae fauna. In addition, Bologna [5], gave 56 species belonging to 12 genera from Turkey. Öncüer [6] listed 91 species, including new records for Turkey. Nizamlıoğlu (1964), Özer and Duran (1968), Özbek (1979), Giray (1985), and Yıldırım and Özbek (1992) mentioned some species of Meloidae as agricultural pests in Turkey [7]. Lodos [8] gave general information (including life cycle and some agricultural pest species) about this family. Özbek and Szaloki [7] examined more than 4000 specimens, which were collected 1970-1996, and 147 species belonging to 2 subfamilies and 17 genera in Turkey. There are three MSc. thesis' on the family Meloidae in Turkey [911]. According to recent literature, the Meloidae fauna of Turkey is represented by 2 subfamilies, 23 genera and 177 species (including 27 endemic species) [12-18].

The main purpose of this study is to determine the faunisticcomposition of the family Meloidae in Ankara province. Escherich [19] stated that Ankara Meloidae fauna is enormously rich and there are more than 40 species. In accordance with this report, we totally detected 43 species belonging to 2 subfamilies and 15 genera from Ankara province.

\section{Study area}

Ankara Province has an area of 26,897 $\mathrm{km}^{2}$ and 25 districts (Altındağ, Çankaya, Mamak, Keçiören, Sincan, Yenimahalle, Akyurt, Beypazarı, Çamlıdere, Çubuk, Elmadağ, Etimesgut, Evren, Kahramankazan, Gölbaşı, Balâ, Ayaş,
Güdül, Haymana, Kalecik, Kızılcahamam, Nallıhan, Polatlı, Pursaklar and Şereflikoçhisar). Most of Ankara's area is located in the Central Anatolian region and the remaining part is located in the Black Sea region of Turkey. It is also located at the intersection of four geographical sections of Turkey (Konya Section, Upper Sakarya Section, Middle Kızılırmak Section, Western Black Sea Section) [20] (Figure 1). Terrestrial climate conditions are dominated in study area. Climatic differences are observed throughout the province; Its south part has the distinct steppe climate, which is the clear features of the Central Anatolia climate, and the temperate and rainy effect of the Black Sea climate are observed in the North. Due to these characteristics, Ankara could be accepted as an intersection area having the characteristics of terrestrial climate [21].

\section{MATERIALS and METHODS}

Material and methods of this study consist of three main phases. These are field studies for specimen collecting, laboratory studies for species determining of collected specimens and analyses of all collected data.

\section{Field studies}

In this study, 3301 adult specimens were collected from 548 locations in 69 days (33 days between May and October 2018 and 36 days between April and October 2019) in Ankara (Figure 1). Specimens were collected from various habitats and Meloidae specimens were found from some habitats such as river-stream edges, open forest areas, road and field edges, meadows. Specimens were collected using insect net and aspirator. Details of the locations with examples are shown in Table 1.

\section{Laboratory studies}

3301 adult specimens collected by field studies were examined for this study, and were identified at subfamily and genus, and then male and female specimens were separated, and species were identified. Identification of species was made with taxonomic keys and species descriptions in the related literature $[3,5,9-11$, 15-17, 24-42]. The male genital organs were dissected and compared with the drawings in the current literature for confirmation of species diagnosis.

\section{Data analysis}

In faunistic evaluations, species diversities of the districts and Turkey's geographical sections, which are lo- 


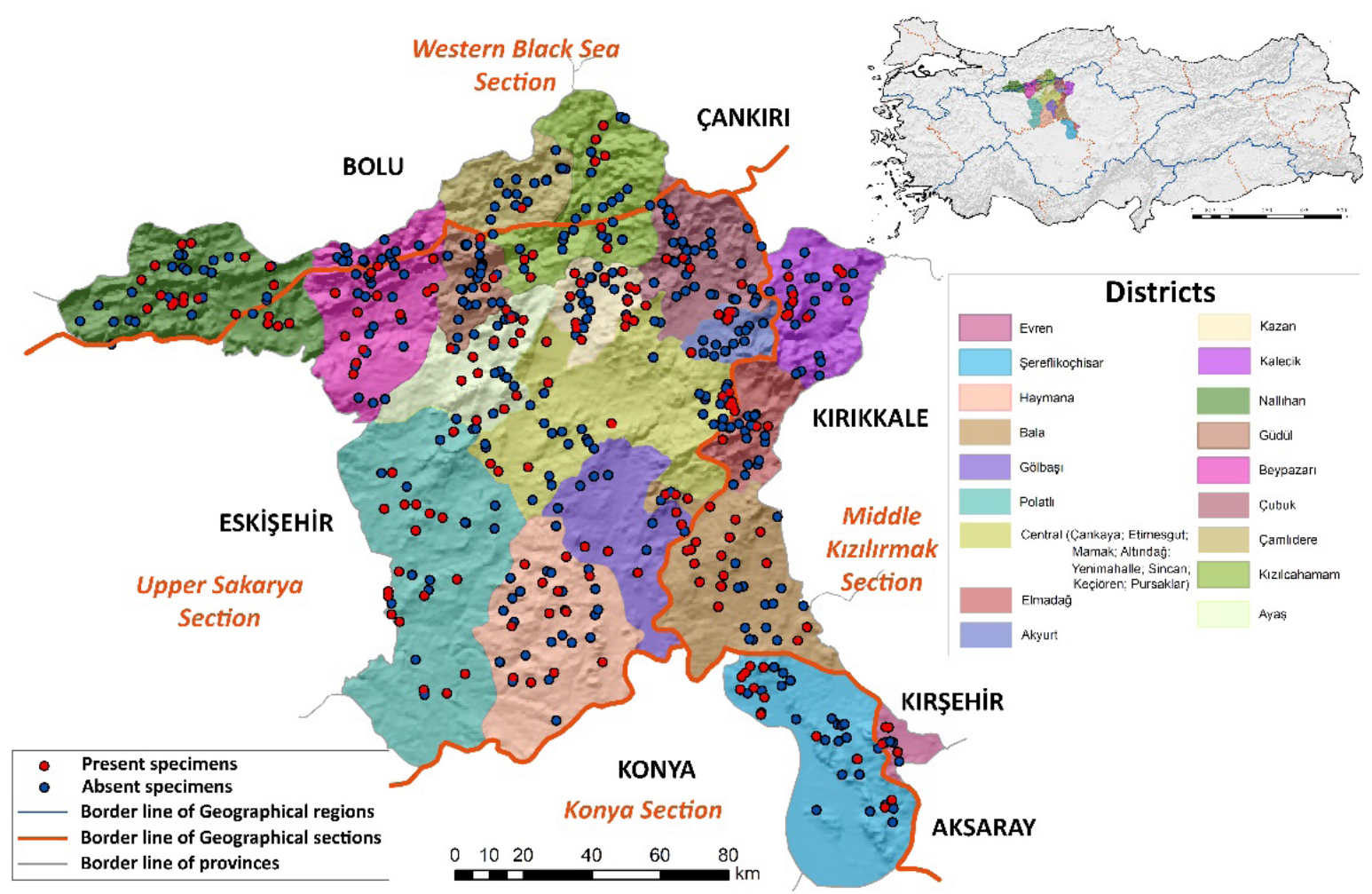

Figure 1. Map of Ankara province (districts are shown in different colors) and collecting localities with presence/absence of specimens (Districts, province, geographical regions and sections data was taken from the Turkey General Directorate of Mapping [22] and designed with ArcGIS Pro [23]).

cated within the boundaries of Ankara province, were evaluated using the Brillouin diversity index [43]. Brillouin diversity index, which is recommended and used in diversity calculations, in cases where ecological randomness is not fully achieved and in studies on collection materials, is considered to be more suitable for this study than other diversity indices. This analysis was made using the software of Past 3.25 [44]. The districts where the urbanization is intense and around the center (Altındağ, Çankaya, Etimesgut, Keçiören, Mamak, Pursaklar, Sincan, Yenimahalle) are evaluated as the Central district. Faunal similarities between Meloidae fauna were determined in this study and faunistic data in the existing literature of the Central Anatolian Region were compared with Baroni-Urbani Büser index [45]. The reason for chosing that it is considered as a better comparison indicator than Sørensen and Jaccard indexes with its evaluation both of the species found in both fauna and the species not found in both fauna. This analysis was done using MVSP 3.21 (MultiVariate Statistical Package 3.21) software [46].

With this study, Teratolytta gentilis (Frivaldszky, 1877), which was determined from Ankara and its detailed locality information was unknown until this study, was not included in the evaluations requiring locality information (collecting coordinate and district).

\section{RESULTS}

In this study, 3301 specimens were collected. 43 species belonging to fifteen genera and two subfamilies were detected during field studies. Code of collecting localities and the number of specimens of species are shown in Table 2. It is noteworthy that the genus Mylabris has the largest amount of species (10 species) following by Cerocoma (6 species), Alosimus and Hycleus (4 species), Meloe, Euzonitis and Zonitis (3 species), Teratolytta, Stenodera (2 species) and Lydus, Oenas, Lytta, Actenodia, Nemognatha, Stenoria (one species each) (Figure 2).

The largest amount of species among the detected species were collected from Bala district (20 species, 631 specimens). This district is followed by Polatlı (18 species, 343 specimens), Haymana (16 species, 389 specimens), Central (15 species), Şereflikoçhisar (13 species), Ayaş, Kazan and Nallıhan (12 species). According to the Brillouin diversity index, species diversity was found the highest in the Central district and later in the Ayaş dis- 
Table 1. Details of collecting localities.

\begin{tabular}{|c|c|c|c|c|c|c|c|c|c|}
\hline \multirow{2}{*}{$\begin{array}{l}\text { Loc. } \\
\text { Code }\end{array}$} & \multirow{2}{*}{ Date } & \multicolumn{2}{|c|}{ Coordinates } & \multirow{2}{*}{$\begin{array}{l}\text { Alti- } \\
\text { tude } \\
(\mathrm{m})\end{array}$} & \multirow{2}{*}{$\begin{array}{l}\text { Loc. } \\
\text { Code }\end{array}$} & \multirow{2}{*}{ Date } & \multicolumn{2}{|c|}{ Coordinates } & \multirow{2}{*}{$\begin{array}{c}\text { Altitude } \\
\text { (m) }\end{array}$} \\
\hline & & Latitude (N) & Longitude (E) & & & & Latitude (N) & Longitude (E) & \\
\hline 1 & 07.04 .2018 & $39^{\circ} 52^{\prime} 08.00^{\prime \prime}$ & $32^{\circ} 44^{\prime} 03.00^{\prime \prime}$ & 970.0 & 34 & 26.06 .2018 & $39^{\circ} 41^{\prime} 17.53^{\prime \prime}$ & $32^{\circ} 55^{\prime} 11.78^{\prime \prime}$ & 1256.7 \\
\hline 2 & 13.05 .2018 & $39^{\circ} 44^{\prime} 17.54^{\prime \prime}$ & $32^{\circ} 20^{\prime} 50.42^{\prime \prime}$ & 754.7 & 35 & 26.06 .2018 & $39^{\circ} 41^{\prime} 18.71^{\prime \prime}$ & $32^{\circ} 57^{\prime} 16.32^{\prime \prime}$ & 1174.6 \\
\hline 3 & 13.05 .2018 & $39^{\circ} 45^{\prime} 28.18^{\prime \prime}$ & $32^{\circ} 19^{\prime} 13.55^{\prime \prime}$ & 787.4 & 36 & 26.06 .2018 & $39^{\circ} 36^{\prime} 38.57^{\prime \prime}$ & $32^{\circ} 58^{\prime} 38.98^{\prime \prime}$ & 1018.6 \\
\hline 4 & 13.05 .2018 & $39^{\circ} 50^{\prime} 20.89^{\prime \prime}$ & $32^{\circ} 11^{\prime} 29.50^{\prime \prime}$ & 861.5 & 37 & 26.06 .2018 & $39^{\circ} 33^{\prime} 51.62^{\prime \prime}$ & $33^{\circ} 01^{\prime} 39.58^{\prime \prime}$ & 1019 \\
\hline 5 & 13.05 .2018 & $39^{\circ} 38^{\prime} 32.35^{\prime \prime}$ & $32^{\circ} 01^{\prime} 51.22^{\prime \prime}$ & 727.5 & 38 & 26.06 .2018 & $39^{\circ} 30^{\prime} 46.11^{\prime \prime}$ & $33^{\circ} 16^{\prime} 12.30^{\prime \prime}$ & 963.7 \\
\hline 6 & 16.05 .2018 & $40^{\circ} 04^{\prime} 41.94^{\prime \prime}$ & $32^{\circ} 23^{\prime} 13.38^{\prime \prime}$ & 1097.5 & 39 & 26.06 .2018 & $39^{\circ} 20^{\prime} 42.81^{\prime \prime}$ & $33^{\circ} 24^{\prime} 40.69^{\prime \prime}$ & 1034.1 \\
\hline 7 & 18.05 .2018 & $39^{\circ} 10^{\prime} 56.70^{\prime \prime}$ & $33^{\circ} 14^{\prime} 04.86^{\prime \prime}$ & 981.2 & 40 & 26.06 .2018 & $39^{\circ} 39^{\prime} 31.59^{\prime \prime}$ & $33^{\circ} 04^{\prime} 23.44^{\prime \prime}$ & 968.6 \\
\hline 8 & 18.05 .2018 & $39^{\circ} 05^{\prime} 06.54^{\prime \prime}$ & $33^{\circ} 40^{\prime} 58.56^{\prime \prime}$ & 931.9 & 41 & 27.06 .2018 & $39^{\circ} 43^{\prime} 06.06^{\prime \prime}$ & $33^{\circ} 09^{\prime} 07.57^{\prime \prime}$ & 915.4 \\
\hline 9 & 19.05 .2018 & $40^{\circ} 11^{\prime} 27.72^{\prime \prime}$ & $31^{\circ} 54^{\prime} 51.36^{\prime \prime}$ & 757.7 & 42 & 27.06 .2018 & $40^{\circ} 04^{\prime} 59.28^{\prime \prime}$ & $32^{\circ} 30^{\prime} 03.86^{\prime \prime}$ & 911.2 \\
\hline 10 & 19.05 .2018 & $40^{\circ} 16^{\prime} 06.90^{\prime \prime}$ & $31^{\circ} 54^{\prime} 48.06^{\prime \prime}$ & 1048.6 & 43 & 27.06 .2018 & $40^{\circ} 08^{\prime} 06.72^{\prime \prime}$ & $32^{\circ} 35^{\prime} 53.30^{\prime \prime}$ & 856 \\
\hline 11 & 19.05 .2018 & $40^{\circ} 03^{\prime} 33.22^{\prime \prime}$ & $32^{\circ} 10^{\prime} 23.67^{\prime \prime}$ & 606.1 & 44 & 27.06.2018 & $40^{\circ} 12^{\prime} 16.24^{\prime \prime}$ & $32^{\circ} 34^{\prime} 41.56^{\prime \prime}$ & 1087.6 \\
\hline 12 & 23.05 .2018 & $39^{\circ} 58^{\prime} 31.80^{\prime \prime}$ & $32^{\circ} 30^{\prime} 35.28^{\prime \prime}$ & 800.6 & 45 & 27.06 .2018 & $40^{\circ} 14^{\prime} 28.67^{\prime \prime}$ & $32^{\circ} 36^{\prime} 47.18^{\prime \prime}$ & 1074.4 \\
\hline 13 & 23.05 .2018 & $39^{\circ} 56^{\prime} 19.98^{\prime \prime}$ & $32^{\circ} 24^{\prime} 09.60^{\prime \prime}$ & 993.7 & 46 & 27.06 .2018 & $40^{\circ} 15^{\prime} 55.28^{\prime \prime}$ & $32^{\circ} 35^{\prime} 28.78^{\prime \prime}$ & 1269.6 \\
\hline 14 & 23.05 .2018 & $39^{\circ} 54^{\prime} 11.22^{\prime \prime}$ & $32^{\circ} 21^{\prime} 44.04^{\prime \prime}$ & 865.3 & 47 & 28.06 .2018 & $39^{\circ} 57^{\prime} 02.54^{\prime \prime}$ & $33^{\circ} 06^{\prime} 28.03^{\prime \prime}$ & 1128.4 \\
\hline 15 & 23.05 .2018 & $40^{\circ} 04^{\prime} 54.18^{\prime \prime}$ & $32^{\circ} 19^{\prime} 17.52^{\prime \prime}$ & 941.2 & 48 & 28.06 .2018 & $39^{\circ} 55^{\prime} 36.83^{\prime \prime}$ & $33^{\circ} 07^{\prime} 54.31^{\prime \prime}$ & 1201.3 \\
\hline 16 & 30.05 .2018 & $40^{\circ} 03^{\prime} 50.46^{\prime \prime}$ & $32^{\circ} 59^{\prime} 50.52^{\prime \prime}$ & 971 & 49 & 28.06 .2018 & $39^{\circ} 52^{\prime} 22.24^{\prime \prime}$ & $33^{\circ} 13^{\prime} 36.31^{\prime \prime}$ & 1149.1 \\
\hline 17 & 30.05 .2018 & $40^{\circ} 09^{\prime} 58.68^{\prime \prime}$ & $33^{\circ} 19^{\prime} 14.28^{\prime \prime}$ & 1088 & 50 & 28.06 .2018 & $39^{\circ} 49^{\prime} 54.72^{\prime \prime}$ & $33^{\circ} 15^{\prime} 24.13^{\prime \prime}$ & 876.1 \\
\hline 18 & 30.05 .2018 & $40^{\circ} 16^{\prime} 04.32^{\prime \prime}$ & $33^{\circ} 19^{\prime} 37.62^{\prime \prime}$ & 995.9 & 51 & 29.06 .2018 & $39^{\circ} 37^{\prime} 47.94^{\prime \prime}$ & $31^{\circ} 57^{\prime} 43.19^{\prime \prime}$ & 688.6 \\
\hline 19 & 2.06 .2018 & $39^{\circ} 52^{\prime} 21.60^{\prime \prime}$ & $32^{\circ} 43^{\prime} 46.93^{\prime \prime}$ & 1012 & 52 & 29.06.2018 & $39^{\circ} 23^{\prime} 58.69^{\prime \prime}$ & $31^{\circ} 59^{\prime} 07.41^{\prime \prime}$ & 711.2 \\
\hline 20 & 11.06 .2018 & $40^{\circ} 11^{\prime} 06.56^{\prime \prime}$ & $32^{\circ} 50^{\prime} 19.27^{\prime \prime}$ & 1168.5 & 53 & 29.06.2018 & $39^{\circ} 21^{\prime} 08.21^{\prime \prime}$ & $31^{\circ} 59^{\prime} 56.36^{\prime \prime}$ & 723.5 \\
\hline 21 & 12.06 .2018 & $39^{\circ} 32^{\prime} 44.14^{\prime \prime}$ & $32^{\circ} 38^{\prime} 59.89^{\prime \prime}$ & 1146.6 & 54 & 29.06.2018 & $39^{\circ} 08^{\prime} 56.37^{\prime \prime}$ & $32^{\circ} 11^{\prime} 42.69^{\prime \prime}$ & 852.3 \\
\hline 22 & 12.06 .2018 & $39^{\circ} 28^{\prime} 06.77^{\prime \prime}$ & $32^{\circ} 40^{\prime} 07.52^{\prime \prime}$ & 1223.5 & 55 & 29.06 .2018 & $39^{\circ} 12^{\prime} 04.41^{\prime \prime}$ & $32^{\circ} 15^{\prime} 17.23^{\prime \prime}$ & 803.4 \\
\hline 23 & 12.06 .2018 & $39^{\circ} 14^{\prime} 31.70^{\prime \prime}$ & $32^{\circ} 43^{\prime} 08.45^{\prime \prime}$ & 1144.8 & 56 & 29.06.2018 & $39^{\circ} 27^{\prime} 00.18^{\prime \prime}$ & $32^{\circ} 13^{\prime} 03.04^{\prime \prime}$ & 998 \\
\hline 24 & 12.06 .2018 & $39^{\circ} 12^{\prime} 38.92^{\prime \prime}$ & $32^{\circ} 33^{\prime} 21.57^{\prime \prime}$ & 1279.8 & 57 & 10.07.2018 & $39^{\circ} 32^{\prime} 06.69^{\prime \prime}$ & $32^{\circ} 43^{\prime} 46.40^{\prime \prime}$ & 1062 \\
\hline 25 & 12.06 .2018 & $39^{\circ} 28^{\prime} 48.96^{\prime \prime}$ & $32^{\circ} 49^{\prime} 55.19^{\prime \prime}$ & 1294.8 & 58 & 19.07.2018 & $39^{\circ} 03^{\prime} 29.38^{\prime \prime}$ & $33^{\circ} 26^{\prime} 58.76^{\prime \prime}$ & 1077.4 \\
\hline 26 & 13.06 .2018 & $40^{\circ} 20^{\prime} 01.32^{\prime \prime}$ & $32^{\circ} 42^{\prime} 06.48^{\prime \prime}$ & 971 & 59 & 19.07 .2018 & $39^{\circ} 02^{\prime} 45.90^{\prime \prime}$ & $33^{\circ} 31^{\prime} 30.30^{\prime \prime}$ & 1068.4 \\
\hline 27 & 25.06 .2018 & $40^{\circ} 06^{\prime} 37.80^{\prime \prime}$ & $31^{\circ} 36^{\prime} 54.84^{\prime \prime}$ & 475.5 & 60 & 19.07.2018 & $38^{\circ} 59^{\prime} 58.20^{\prime \prime}$ & $33^{\circ} 35^{\prime} 24.90^{\prime \prime}$ & 1046.1 \\
\hline 28 & 25.06 .2018 & $40^{\circ} 12^{\prime} 33.29^{\prime \prime}$ & $31^{\circ} 33^{\prime} 29.18^{\prime \prime}$ & 975.5 & 61 & 19.07.2018 & $39^{\circ} 02^{\prime} 23.30^{\prime \prime}$ & $33^{\circ} 40^{\prime} 18.00^{\prime \prime}$ & 1355.8 \\
\hline 29 & 25.06 .2018 & $40^{\circ} 15^{\prime} 26.86^{\prime \prime}$ & $31^{\circ} 32^{\prime} 30.80^{\prime \prime}$ & 1361.3 & 62 & 19.07.2018 & $39^{\circ} 01^{\prime} 08.00^{\prime \prime}$ & $33^{\circ} 43^{\prime} 30.30^{\prime \prime}$ & 1040.8 \\
\hline 30 & 25.06 .2018 & $40^{\circ} 16^{\prime} 51.22^{\prime \prime}$ & $31^{\circ} 27^{\prime} 17.66^{\prime \prime}$ & 922 & 63 & 19.07.2018 & $38^{\circ} 53^{\prime} 34.10^{\prime \prime}$ & $33^{\circ} 42^{\prime} 28.60^{\prime \prime}$ & 1104.1 \\
\hline 31 & 25.06 .2018 & $40^{\circ} 09^{\prime} 58.11^{\prime \prime}$ & $31^{\circ} 17^{\prime} 59.70^{\prime \prime}$ & 729.6 & 64 & 19.07.2018 & $39^{\circ} 12^{\prime} 40.40^{\prime \prime}$ & $33^{\circ} 11^{\prime} 31.70^{\prime \prime}$ & 1083.4 \\
\hline 32 & 25.06 .2018 & $40^{\circ} 09^{\prime} 14.63^{\prime \prime}$ & $31^{\circ} 14^{\prime} 58.08^{\prime \prime}$ & 1069.5 & 65 & 20.07 .2018 & $40^{\circ} 08^{\prime} 44.10^{\prime \prime}$ & $31^{\circ} 50^{\prime} 54.50^{\prime \prime}$ & 649.6 \\
\hline 33 & 25.06 .2018 & $40^{\circ} 08^{\prime} 02.94^{\prime \prime}$ & $31^{\circ} 07^{\prime} 35.28^{\prime \prime}$ & 686.2 & 66 & 20.07.2018 & $40^{\circ} 11^{\prime} 39.20^{\prime \prime}$ & $31^{\circ} 46^{\prime} 10.50^{\prime \prime}$ & 866.7 \\
\hline
\end{tabular}


Table 1. Details of collecting localities. (Continued)

\begin{tabular}{|c|c|c|c|c|c|c|c|c|c|}
\hline \multirow{2}{*}{$\begin{array}{l}\text { Loc. } \\
\text { Code }\end{array}$} & \multirow{2}{*}{ Date } & \multicolumn{2}{|c|}{ Coordinates } & \multirow{2}{*}{$\begin{array}{l}\text { Alti- } \\
\text { tude } \\
\text { (m) }\end{array}$} & \multirow{2}{*}{$\begin{array}{l}\text { Loc. } \\
\text { Code }\end{array}$} & \multirow{2}{*}{ Date } & \multicolumn{2}{|c|}{ Coordinates } & \multirow{2}{*}{$\begin{array}{l}\text { Altitude } \\
\qquad(\mathrm{m})\end{array}$} \\
\hline & & Latitude (N) & Longitude (E) & & & & Latitude (N) & Longitude (E) & \\
\hline 67 & 20.07.2018 & $40^{\circ} 04^{\prime} 53.10^{\prime \prime}$ & $31^{\circ} 48^{\prime} 31.10^{\prime \prime}$ & 498.1 & 100 & 05.08 .2018 & $40^{\circ} 11^{\prime} 44.50^{\prime \prime}$ & $32^{\circ} 46^{\prime} 33.70^{\prime \prime}$ & 1077 \\
\hline 68 & 20.07 .2018 & $40^{\circ} 02^{\prime} 50.90^{\prime \prime}$ & $31^{\circ} 52^{\prime} 20.10^{\prime \prime}$ & 641.5 & 101 & 05.08 .2018 & $40^{\circ} 08^{\prime} 39.60^{\prime \prime}$ & $32^{\circ} 47^{\prime} 53.70^{\prime \prime}$ & 1252.3 \\
\hline 69 & 20.07.2018 & $40^{\circ} 00^{\prime} 42.20^{\prime \prime}$ & $31^{\circ} 51^{\prime} 00.90^{\prime \prime}$ & 922.8 & 102 & 28.08 .2018 & $39^{\circ} 45^{\prime} 04.97^{\prime \prime}$ & $32^{\circ} 26^{\prime} 58.01^{\prime \prime}$ & 816.9 \\
\hline 70 & 20.07.2018 & $39^{\circ} 58^{\prime} 56.70^{\prime \prime}$ & $31^{\circ} 50^{\prime} 29.40^{\prime \prime}$ & 1023.5 & 103 & 20.04 .2019 & $39^{\circ} 03^{\prime 2} 29.22^{\prime \prime}$ & $33^{\circ} 26^{\prime} 54.96^{\prime \prime}$ & 1065.7 \\
\hline 71 & 21.07.2018 & $39^{\circ} 59^{\prime} 43.10^{\prime \prime}$ & $32^{\circ} 16^{\prime} 06.10^{\prime \prime}$ & 794.8 & 104 & 22.04 .2019 & $39^{\circ} 52^{\prime} 13.80^{\prime \prime}$ & $32^{\circ} 16^{\prime} 06.72^{\prime \prime}$ & 758.6 \\
\hline 72 & 21.07 .2018 & $39^{\circ} 58^{\prime} 27.50^{\prime \prime}$ & $32^{\circ} 12^{\prime} 44.00^{\prime \prime}$ & 828.5 & 105 & 22.04 .2019 & $40^{\circ} 02^{\prime} 16.38^{\prime \prime}$ & $32^{\circ} 14^{\prime} 59.34^{\prime \prime}$ & 706.6 \\
\hline 73 & 21.07 .2018 & $40^{\circ} 03^{\prime} 24.70^{\prime \prime}$ & $32^{\circ} 11^{\prime} 10.80^{\prime \prime}$ & 612.9 & 106 & 07.05 .2019 & $40^{\circ} 06^{\prime} 06.87^{\prime \prime}$ & 31³4'38.93" & 561.3 \\
\hline 74 & 21.07.2018 & $40^{\circ} 21^{\prime} 02.90^{\prime \prime}$ & $32^{\circ} 15^{\prime} 46.70^{\prime \prime}$ & 1346 & 107 & 07.05.2019 & $40^{\circ} 06^{\prime} 25.45^{\prime \prime}$ & 31³2'31.19" & 589 \\
\hline 75 & 21.07.2018 & $40^{\circ} 13^{\prime} 19.60^{\prime \prime}$ & $32^{\circ} 17^{\prime} 09.40^{\prime \prime}$ & 824.7 & 108 & 07.05.2019 & $40^{\circ} 07^{\prime} 35.89^{\prime \prime}$ & $31^{\circ} 32^{\prime} 41.15^{\prime \prime}$ & 617.8 \\
\hline 76 & 21.07.2018 & $40^{\circ} 14^{\prime} 54.20^{\prime \prime}$ & $32^{\circ} 18^{\prime} 49.50^{\prime \prime}$ & 949.6 & 109 & 23.05.2019 & $39^{\circ} 40^{\prime} 43.98^{\prime \prime}$ & $32^{\circ} 59^{\prime} 48.54^{\prime \prime}$ & 1050.1 \\
\hline 77 & 22.07.2018 & $40^{\circ} 09^{\prime} 06.00^{\prime \prime}$ & $33^{\circ} 07^{\prime} 03.50^{\prime \prime}$ & 1217 & 110 & 23.05 .2019 & $39^{\circ} 38^{\prime} 29.94^{\prime \prime}$ & $32^{\circ} 57^{\prime} 30.90^{\prime \prime}$ & 1111.1 \\
\hline 78 & 22.07 .2018 & $40^{\circ} 10^{\prime} 23.30^{\prime \prime}$ & $33^{\circ} 08^{\prime} 03.60^{\prime \prime}$ & 1332.8 & 111 & 23.05.2019 & $39^{\circ} 36^{\prime} 29.40^{\prime \prime}$ & $32^{\circ} 58^{\prime} 43.56^{\prime \prime}$ & 1014 \\
\hline 79 & 22.07 .2018 & $40^{\circ} 09^{\prime} 45.00^{\prime \prime}$ & $33^{\circ} 23^{\prime} 38.10^{\prime \prime}$ & 983.9 & 112 & 23.05 .2019 & $39^{\circ} 32^{\prime} 29.34^{\prime \prime}$ & $32^{\circ} 59^{\prime} 52.56^{\prime \prime}$ & 1197.8 \\
\hline 80 & 22.07 .2018 & $40^{\circ} 15^{\prime} 56.30^{\prime \prime}$ & $33^{\circ} 23^{\prime} 53.20^{\prime \prime}$ & 939.3 & 113 & 23.05 .2019 & $39^{\circ} 32^{\prime} 38.16^{\prime \prime}$ & $33^{\circ} 00^{\prime} 39.90^{\prime \prime}$ & 1165.5 \\
\hline 81 & 22.07 .2018 & $40^{\circ} 12^{\prime} 30.40^{\prime \prime}$ & $33^{\circ} 31^{\prime} 50.00^{\prime \prime}$ & 673.6 & 114 & 23.05 .2019 & $39^{\circ} 37^{\prime} 33.78^{\prime \prime}$ & $33^{\circ} 09^{\prime} 38.58^{\prime \prime}$ & 1089.8 \\
\hline 82 & 23.07 .2018 & $40^{\circ} 14^{\prime} 48.10^{\prime \prime}$ & $33^{\circ} 09^{\prime} 59.70^{\prime \prime}$ & 1005.3 & 115 & 24.05 .2019 & $40^{\circ} 26^{\prime} 04.10^{\prime \prime}$ & $32^{\circ} 24^{\prime} 09.40^{\prime \prime}$ & 1048.2 \\
\hline 83 & 23.07.2018 & $40^{\circ} 25^{\prime} 13.80^{\prime \prime}$ & $32^{\circ} 55^{\prime} 07.80^{\prime \prime}$ & 1535.9 & 116 & 25.05 .2019 & $40^{\circ} 17^{\prime} 09.20^{\prime \prime}$ & $32^{\circ} 59^{\prime} 16.20^{\prime \prime}$ & 1213 \\
\hline 84 & 24.07.2018 & $40^{\circ} 15^{\prime} 07.00^{\prime \prime}$ & $32^{\circ} 26^{\prime} 23.50^{\prime \prime}$ & 1268 & 117 & 26.05 .2019 & $39^{\circ} 52^{\prime} 24.00^{\prime \prime}$ & $33^{\circ} 15^{\prime} 56.20^{\prime \prime}$ & 944 \\
\hline 85 & 24.07 .2018 & $40^{\circ} 17^{\prime} 56.90^{\prime \prime}$ & $32^{\circ} 25^{\prime} 01.30^{\prime \prime}$ & 773.5 & 118 & 18.06 .2019 & $40^{\circ} 15^{\prime} 03.30^{\prime \prime}$ & $31^{\circ} 53^{\prime} 23.90^{\prime \prime}$ & 1212.8 \\
\hline 86 & 25.07 .2018 & $39^{\circ} 31^{\prime} 03.80^{\prime \prime}$ & $32^{\circ} 35^{\prime} 13.10^{\prime \prime}$ & 1226.9 & 119 & 19.06.2019 & $39^{\circ} 36^{\prime} 41.20^{\prime \prime}$ & $32^{\circ} 09^{\prime} 44.00^{\prime \prime}$ & 930.2 \\
\hline 87 & 25.07 .2018 & $39^{\circ} 29^{\prime} 39.70^{\prime \prime}$ & $32^{\circ} 26^{\prime} 46.20^{\prime \prime}$ & 969.2 & 120 & 19.06 .2019 & $39^{\circ} 37^{\prime} 17.90^{\prime \prime}$ & $32^{\circ} 07^{\prime} 06.20^{\prime \prime}$ & 957.4 \\
\hline 88 & 25.07.2018 & $39^{\circ} 11^{\prime} 40.30^{\prime \prime}$ & $32^{\circ} 25^{\prime} 06.80^{\prime \prime}$ & 1038.8 & 121 & 19.06.2019 & $39^{\circ} 38^{\prime} 38.50^{\prime \prime}$ & $32^{\circ} 04^{\prime} 14.70^{\prime \prime}$ & 784.4 \\
\hline 89 & 25.07 .2018 & $39^{\circ} 22^{\prime} 01.50^{\prime \prime}$ & $32^{\circ} 32^{\prime} 03.50^{\prime \prime}$ & 1245 & 122 & 19.06.2019 & $39^{\circ} 43^{\prime} 33.60^{\prime \prime}$ & $31^{\circ} 59^{\prime} 08.70^{\prime \prime}$ & 677.7 \\
\hline 90 & 26.07 .2018 & $39^{\circ} 31^{\prime} 48.80^{\prime \prime}$ & $33^{\circ} 06^{\prime} 55.20^{\prime \prime}$ & 1284.2 & 123 & 19.06.2019 & $39^{\circ} 34^{\prime} 31.10^{\prime \prime}$ & $32^{\circ} 04^{\prime} 19.80^{\prime \prime}$ & 800.6 \\
\hline 91 & 26.07 .2018 & $39^{\circ} 30^{\prime} 27.00^{\prime \prime}$ & $33^{\circ} 01^{\prime} 25.40^{\prime \prime}$ & 1281.2 & 124 & 20.06 .2019 & $40^{\circ} 07^{\prime} 18.50^{\prime \prime}$ & $32^{\circ} 36^{\prime} 06.60^{\prime \prime}$ & 848.8 \\
\hline 92 & 26.07 .2018 & $39^{\circ} 27^{\prime} 32.60^{\prime \prime}$ & $33^{\circ} 02^{\prime} 36.50^{\prime \prime}$ & 1125.8 & 125 & 21.06.2019 & $40^{\circ} 10^{\prime} 15.00^{\prime \prime}$ & $33^{\circ} 19^{\prime} 48.90^{\prime \prime}$ & 1087 \\
\hline 93 & 26.07.2018 & $39^{\circ} 26^{\prime} 55.40^{\prime \prime}$ & $33^{\circ} 07^{\prime} 06.30^{\prime \prime}$ & 962.6 & 126 & 21.06 .2019 & $40^{\circ} 12^{\prime} 13.50^{\prime \prime}$ & $33^{\circ} 20^{\prime} 34.80^{\prime \prime}$ & 1039.9 \\
\hline 94 & 26.07.2018 & $39^{\circ} 35^{\prime} 40.90^{\prime \prime}$ & $33^{\circ} 14^{\prime} 48.90^{\prime \prime}$ & 1129.7 & 127 & 21.06.2019 & $40^{\circ} 13^{\prime} 24.20^{\prime \prime}$ & $33^{\circ} 20^{\prime} 34.80^{\prime \prime}$ & 1032.8 \\
\hline 95 & 26.07 .2018 & $39^{\circ} 34^{\prime} 42.80^{\prime \prime}$ & $33^{\circ} 08^{\prime} 30.70^{\prime \prime}$ & 1164.2 & 128 & 21.06 .2019 & $40^{\circ} 12^{\prime} 29.70^{\prime \prime}$ & $33^{\circ} 31^{\prime} 51.10^{\prime \prime}$ & 674 \\
\hline 96 & 04.08 .2018 & $40^{\circ} 14^{\prime} 55.40^{\prime \prime}$ & $31^{\circ} 08^{\prime} 47.90^{\prime \prime}$ & 1386.1 & 129 & 27.06.2019 & $40^{\circ} 06^{\prime} 16.00^{\prime \prime}$ & $32^{\circ} 24^{\prime} 34.00^{\prime \prime}$ & 1033 \\
\hline 97 & 04.08 .2018 & $40^{\circ} 12^{\prime} 34.70^{\prime \prime}$ & $31^{\circ} 06^{\prime} 04.90^{\prime \prime}$ & 835.9 & 130 & 27.06.2019 & $40^{\circ} 08^{\prime} 20.40^{\prime \prime}$ & $32^{\circ} 25^{\prime} 07.00^{\prime \prime}$ & 1035.4 \\
\hline 98 & 04.08 .2018 & $40^{\circ} 10^{\prime} 14.60^{\prime \prime}$ & $31^{\circ} 10^{\prime} 16.60^{\prime \prime}$ & 742.6 & 131 & 27.06.2019 & $40^{\circ} 08^{\prime} 35.00^{\prime \prime}$ & $32^{\circ} 22^{\prime} 43.50^{\prime \prime}$ & 884.5 \\
\hline 99 & 04.08 .2018 & $40^{\circ} 07^{\prime} 44.40^{\prime \prime}$ & $31^{\circ} 25^{\prime} 49.50^{\prime \prime}$ & 537.3 & 132 & 27.06.2019 & $40^{\circ} 09^{\prime} 50.10^{\prime \prime}$ & $32^{\circ} 21^{\prime} 35.20^{\prime \prime}$ & 1023.3 \\
\hline
\end{tabular}


Table 1. Details of collecting localities. (Continued)

\begin{tabular}{|c|c|c|c|c|c|c|c|c|c|}
\hline \multirow{2}{*}{$\begin{array}{l}\text { Loc. } \\
\text { Code }\end{array}$} & \multirow{2}{*}{ Date } & \multicolumn{2}{|c|}{ Coordinates } & \multirow{2}{*}{$\begin{array}{l}\text { Alti- } \\
\text { tude } \\
\text { (m) }\end{array}$} & \multirow{2}{*}{$\begin{array}{l}\text { Loc. } \\
\text { Code }\end{array}$} & \multirow{2}{*}{ Date } & \multicolumn{2}{|c|}{ Coordinates } & \multirow{2}{*}{$\begin{array}{l}\text { Altitude } \\
\quad(\mathrm{m})\end{array}$} \\
\hline & & Latitude (N) & Longitude (E) & & & & Latitude (N) & Longitude (E) & \\
\hline 133 & 28.06 .2019 & $39^{\circ} 10^{\prime} 40.50^{\prime \prime}$ & $33^{\circ} 11^{\prime} 19.90^{\prime \prime}$ & 1077.5 & 162 & 26.07.2019 & $40^{\circ} 18^{\prime} 33.80^{\prime \prime}$ & $32^{\circ} 54^{\prime} 21.00^{\prime \prime}$ & 1269 \\
\hline 134 & 28.06 .2019 & 39¹3'21.70" & $33^{\circ} 12^{\prime} 18.40^{\prime \prime}$ & 1198.2 & 163 & 26.07 .2019 & $40^{\circ} 18^{\prime} 47.20^{\prime \prime}$ & $32^{\circ} 57^{\prime} 43.50^{\prime \prime}$ & 1216.7 \\
\hline 135 & 28.06 .2019 & 39¹4'21.90" & $33^{\circ} 13^{\prime} 14.10^{\prime \prime}$ & 1270.3 & 164 & 27.07.2019 & $39^{\circ} 27^{\prime} 55.50^{\prime \prime}$ & $32^{\circ} 01^{\prime} 12.10^{\prime \prime}$ & 733.5 \\
\hline 136 & 28.06 .2019 & $39^{\circ} 14^{\prime} 14.00^{\prime \prime}$ & $33^{\circ} 15^{\prime} 57.70^{\prime \prime}$ & 1307.9 & 165 & 27.07.2019 & $39^{\circ} 24^{\prime} 45.10^{\prime \prime}$ & $31^{\circ} 59^{\prime} 07.50^{\prime \prime}$ & 706.5 \\
\hline 137 & 28.06 .2019 & $39^{\circ} 09^{\prime} 29.60^{\prime \prime}$ & $33^{\circ} 16^{\prime} 16.20^{\prime \prime}$ & 928.9 & 166 & 27.07.2019 & $39^{\circ} 20^{\prime} 00.00^{\prime \prime}$ & $32^{\circ} 01^{\prime} 35.80^{\prime \prime}$ & 730 \\
\hline 138 & 28.06 .2019 & $39^{\circ} 05^{\prime} 03.10^{\prime \prime}$ & $33^{\circ} 41^{\prime} 37.50^{\prime \prime}$ & 895.3 & 167 & 27.07.2019 & 3909'19.70" & $32^{\circ} 07^{\prime} 01.10^{\prime \prime}$ & 908.3 \\
\hline 139 & 29.06.2019 & $39^{\circ} 25^{\prime} 33.80^{\prime \prime}$ & $32^{\circ} 33^{\prime} 45.70^{\prime \prime}$ & 1278.3 & 168 & 27.07.2019 & $39^{\circ} 24^{\prime} 13.50^{\prime \prime}$ & $32^{\circ} 06^{\prime} 30.00^{\prime \prime}$ & 926 \\
\hline 140 & 29.06 .2019 & $39^{\circ} 22^{\prime} 20.20^{\prime \prime}$ & $32^{\circ} 35^{\prime} 29.70^{\prime \prime}$ & 1118.8 & 169 & 28.07.2019 & $40^{\circ} 09^{\prime} 55.30^{\prime \prime}$ & $33^{\circ} 05^{\prime} 22.50^{\prime \prime}$ & 1082.2 \\
\hline 141 & 29.06.2019 & $39^{\circ} 11^{\prime} 00.60^{\prime \prime}$ & $32^{\circ} 28^{\prime} 42.80^{\prime \prime}$ & 1158.8 & 170 & 28.07 .2019 & $40^{\circ} 10^{\prime} 20.90^{\prime \prime}$ & $33^{\circ} 07^{\prime} 25.20^{\prime \prime}$ & 1287.1 \\
\hline 142 & 30.06 .2019 & $40^{\circ} 18^{\prime} 41.80^{\prime \prime}$ & $31^{\circ} 16^{\prime} 03.30^{\prime \prime}$ & 782.8 & 171 & 28.07 .2019 & $40^{\circ} 09^{\prime} 17.70^{\prime \prime}$ & $33^{\circ} 07^{\prime} 23.20^{\prime \prime}$ & 1251.1 \\
\hline 143 & 30.06 .2019 & $40^{\circ} 18^{\prime} 27.20^{\prime \prime}$ & $31^{\circ} 14^{\prime} 10.40^{\prime \prime}$ & 813.4 & 172 & 28.07.2019 & $40^{\circ} 16^{\prime} 50.70^{\prime \prime}$ & $33^{\circ} 30^{\prime} 42.50^{\prime \prime}$ & 819.3 \\
\hline 144 & 30.06 .2019 & $40^{\circ} 10^{\prime} 09.70^{\prime \prime}$ & $31^{\circ} 14^{\prime} 57.40^{\prime \prime}$ & 948.9 & 173 & 28.07.2019 & $40^{\circ} 17^{\prime} 30.30^{\prime \prime}$ & $33^{\circ} 29^{\prime} 47.30^{\prime \prime}$ & 832.2 \\
\hline 145 & 30.06 .2019 & $40^{\circ} 09^{\prime} 14.10^{\prime \prime}$ & $31^{\circ} 13^{\prime} 03.40^{\prime \prime}$ & 945.9 & 174 & 29.07.2019 & $40^{\circ} 08^{\prime} 38.60^{\prime \prime}$ & $31^{\circ} 59^{\prime} 42.60^{\prime \prime}$ & 579.4 \\
\hline 146 & 30.06 .2019 & $40^{\circ} 08^{\prime} 45.70^{\prime \prime}$ & $31^{\circ} 12^{\prime} 37.00^{\prime \prime}$ & 959.2 & 175 & 29.07.2019 & $40^{\circ} 12^{\prime} 18.40^{\prime \prime}$ & $32^{\circ} 05^{\prime} 11.60^{\prime \prime}$ & 695.2 \\
\hline 147 & 23.07.2019 & $40^{\circ} 23^{\prime} 14.90^{\prime \prime}$ & $32^{\circ} 40^{\prime} 43.20^{\prime \prime}$ & 1045.6 & 176 & 29.07 .2019 & $40^{\circ} 12^{\prime} 59.20^{\prime \prime}$ & $32^{\circ} 06^{\prime} 28.50^{\prime \prime}$ & 759.8 \\
\hline 148 & 23.07.2019 & $40^{\circ} 33^{\prime} 43.70^{\prime \prime}$ & $32^{\circ} 39^{\prime} 04.60^{\prime \prime}$ & 1096.2 & 177 & 29.07.2019 & $40^{\circ} 17^{\prime} 35.80^{\prime \prime}$ & $32^{\circ} 06^{\prime} 09.90^{\prime \prime}$ & 868.1 \\
\hline 149 & 23.07 .2019 & $40^{\circ} 34^{\prime} 37.50^{\prime \prime}$ & $32^{\circ} 41^{\prime} 07.80^{\prime \prime}$ & 1233 & 178 & 30.07 .2019 & $40^{\circ} 05^{\prime} 27.20^{\prime \prime}$ & $32^{\circ} 37^{\prime} 12.70^{\prime \prime}$ & 838.9 \\
\hline 150 & 23.07 .2019 & $40^{\circ} 39^{\prime} 24.90^{\prime \prime}$ & $32^{\circ} 40^{\prime} 34.40^{\prime \prime}$ & 1441.4 & 179 & 30.07 .2019 & $40^{\circ} 15^{\prime} 22.50^{\prime \prime}$ & $32^{\circ} 41^{\prime} 35.20^{\prime \prime}$ & 921.3 \\
\hline 151 & 23.07.2019 & $40^{\circ} 37^{\prime} 11.70^{\prime \prime}$ & $32^{\circ} 39^{\prime} 05.90^{\prime \prime}$ & 1165.5 & 180 & 30.07 .2019 & $40^{\circ} 16^{\prime} 24.60^{\prime \prime}$ & $32^{\circ} 45^{\prime} 17.50^{\prime \prime}$ & 1118.5 \\
\hline 152 & 24.07.2019 & $39^{\circ} 57^{\prime} 43.50^{\prime \prime}$ & $33^{\circ} 07^{\prime} 54.30^{\prime \prime}$ & 1115.1 & 181 & 30.07 .2019 & $40^{\circ} 13^{\prime} 59.40^{\prime \prime}$ & $32^{\circ} 46^{\prime} 50.60^{\prime \prime}$ & 926.8 \\
\hline 153 & 24.07.2019 & $39^{\circ} 56^{\prime} 13.70^{\prime \prime}$ & $33^{\circ} 08^{\prime} 26.60^{\prime \prime}$ & 1211.1 & 182 & 30.07 .2019 & $40^{\circ} 09^{\prime} 06.30^{\prime \prime}$ & $32^{\circ} 46^{\prime} 24.90^{\prime \prime}$ & 1273.6 \\
\hline 154 & 24.07 .2019 & $39^{\circ} 55^{\prime} 31.40^{\prime \prime}$ & $33^{\circ} 08^{\prime} 40.60^{\prime \prime}$ & 1241.2 & 183 & 30.07 .2019 & $40^{\circ} 07^{\prime} 43.80^{\prime \prime}$ & $32^{\circ} 46^{\prime} 08.40^{\prime \prime}$ & 1111.9 \\
\hline 155 & 24.07 .2019 & $39^{\circ} 54^{\prime} 44.80^{\prime \prime}$ & $33^{\circ} 09^{\prime} 04.50^{\prime \prime}$ & 1425.6 & 184 & 27.08 .2019 & $39^{\circ} 06^{\prime} 45.40^{\prime \prime}$ & $33^{\circ} 15^{\prime} 36.40^{\prime \prime}$ & 985.2 \\
\hline 156 & 24.07 .2019 & $39^{\circ} 50^{\prime} 08.60^{\prime \prime}$ & $33^{\circ} 06^{\prime} 42.10^{\prime \prime}$ & 1619.4 & 185 & 27.08 .2019 & $38^{\circ} 52^{\prime} 22.90^{\prime \prime}$ & $33^{\circ} 41^{\prime} 04.80^{\prime \prime}$ & 1097.2 \\
\hline 157 & 25.07.2019 & $39^{\circ} 28^{\prime} 38.20^{\prime \prime}$ & $33^{\circ} 07^{\prime} 27.90^{\prime \prime}$ & 1078.1 & 186 & 27.08 .2019 & $38^{\circ} 53^{\prime} 35.50^{\prime \prime}$ & $33^{\circ} 42^{\prime} 27.50^{\prime \prime}$ & 1102.2 \\
\hline 158 & 25.07 .2019 & $39^{\circ} 27^{\prime} 00.30^{\prime \prime}$ & $33^{\circ} 06^{\prime} 55.10^{\prime \prime}$ & 964.3 & 187 & 29.08 .2019 & $39^{\circ} 26^{\prime} 50.90^{\prime \prime}$ & $32^{\circ} 30^{\prime} 30.30^{\prime \prime}$ & 1163.7 \\
\hline 159 & 25.07 .2019 & $39^{\circ} 23^{\prime} 41.50^{\prime \prime}$ & $33^{\circ} 06^{\prime} 31.40^{\prime \prime}$ & 965.3 & 188 & 29.08 .2019 & $39^{\circ} 19^{\prime} 50.60^{\prime \prime}$ & $32^{\circ} 24^{\prime} 28.80^{\prime \prime}$ & 1143.5 \\
\hline 160 & 25.07.2019 & $39^{\circ} 18^{\prime} 35.40^{\prime \prime}$ & $33^{\circ} 22^{\prime} 50.60^{\prime \prime}$ & 836.6 & 189 & 31.08 .2019 & $40^{\circ} 18^{\prime} 40.30^{\prime \prime}$ & $32^{\circ} 26^{\prime} 41.80^{\prime \prime}$ & 783.7 \\
\hline 161 & 26.07.2019 & $40^{\circ} 13^{\prime} 39.00^{\prime \prime}$ & $32^{\circ} 48^{\prime} 10.90^{\prime \prime}$ & 951.9 & & & & & \\
\hline
\end{tabular}

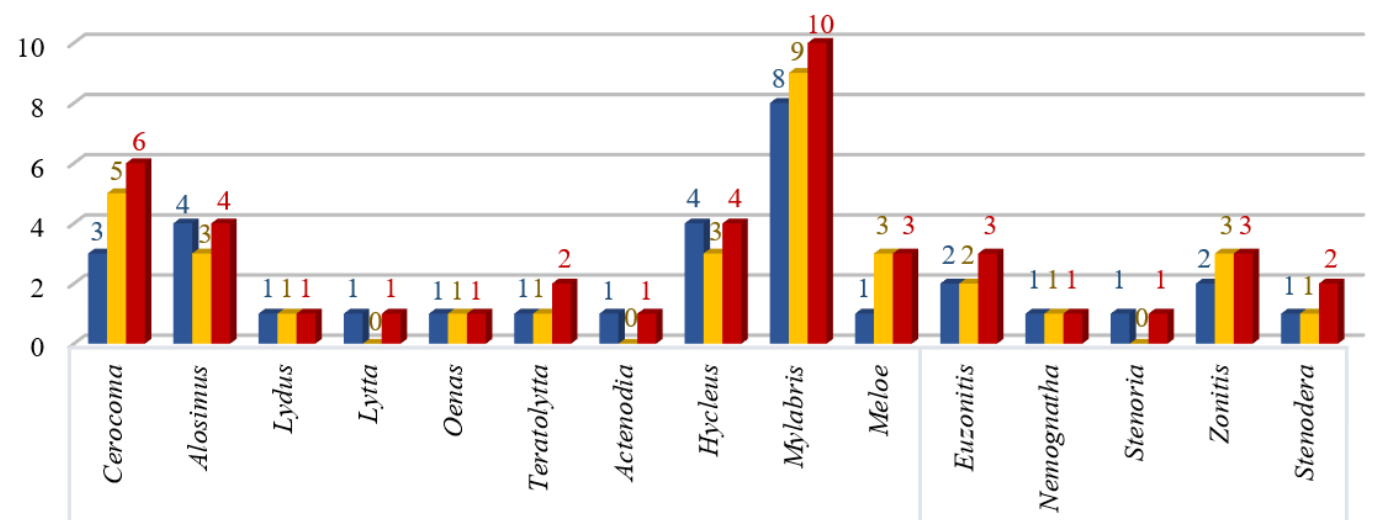

Figure 2. The number of species belonging to subfamilies and genera detected in 2018, 2019 and total. 
trict. Although the number of species is less, as a result of the analysis, it seems that the district of Central and Ayaş exhibited higher species diversity (Table 3).

Ankara Meloidae fauna represents the Upper Sakarya Section, which covers the most areas and the largest amount of species (37 species). The least faunal components of Ankara are detected as parts of the Western Black Sea section and Konya section with 12 species each (Table 3, Figure 3).
Compared to the existing literature, the detected Ankara fauna, 14 species were added with this study and 14 species could not be detected. Species that cannot be detected are shown in Table 4.

Meloidae fauna similarity between the detected in this study and recorded in the current literature was compared (Figure 4, Table 5). Faunistic result of this study is the closest to faunistic data of Ankara in current litera-

Table 2. Male-female specimen numbers of detected species and code of collecting localities.

\begin{tabular}{|c|c|c|}
\hline Species & Number of specimens & Locality Code \\
\hline Cerocoma (s.str.) bernhaueri Pardo Alcaide, 1977 & $3(2 \hat{\sigma}, 1 q)$ & $15,106,114$ \\
\hline Cerocoma (s.str.) dahli Kraatz, 1863 & $2(299)$ & 2 \\
\hline Cerocoma (s.str.) schaefferi (Linnaeus, 1758) & $1(1 \precsim)$ & 151 \\
\hline $\begin{array}{l}\text { Cerocoma (Meloides) longiseta Turco \& Bologna, } \\
2011\end{array}$ & $5(1 \widehat{\jmath}, 4+q)$ & $8,16,22,43,122$ \\
\hline Cerocoma (M.) turcica Pardo Alcaide, 1977 & $1(1 \precsim)$ & 178 \\
\hline Cerocoma (Metacerocoma) ephesica Reitter, 1885 & $8(89+9)$ & $138,163,164$ \\
\hline Alosimus armeniacus (Faldermann, 1837) & $118(72 \hat{\jmath}, 46 q+)$ & $\begin{array}{c}2,3,6,14,16,17,19,20,23,36,105,111,113,116 \\
117,125,129\end{array}$ \\
\hline Alosimus chalybaeus (Tauscher, 1812) & $176(106 \delta \hat{\sigma}, 70+9)$ & $\begin{array}{c}2,3,4,6,7,11,13,14,19,20,21,23,24,27,106,109 \\
110,111,113,115,119,122,124\end{array}$ \\
\hline Alosimus luteus (Waltl, 1838) & $1(1 \precsim)$ & 5 \\
\hline Alosimus marginicollis (Haag-Rutenberg, 1880) & $12(6 \hat{\jmath}, 6$ 우) & 3,113 \\
\hline Lydus turcicus Kaszab, 1952 & $58(36 ふ \hat{\jmath}, 22+q)$ & $24,90,118,120,133,134,136,137,139,152,171$ \\
\hline $\begin{array}{l}\text { Lytta (s.str.) vesicatoria heydeni Haag-Rutenberg, } \\
\qquad 1880\end{array}$ & $1(1+)$ & 9 \\
\hline Oenas crassicornis Illiger, 1800 & $147(68 ふ \hat{\jmath}, 79+9)$ & $\begin{array}{l}41,43,47,50,57,59,60,63,71,72,73,80,139,153 \\
159,161,162,165,169,171,173,176,178,181,185\end{array}$ \\
\hline Teratolytta gentilis (Frivaldszky, 1877) & $1(19)$ & - \\
\hline Teratolytta monticola (Bologna, 2006) & $1(1+)$ & 108 \\
\hline Actenodia confluens (Reiche, 1866) & $8(3 \hat{\jmath}, 5 q+)$ & 55 \\
\hline Hycleus polymorphus (Pallas, 1771) & $12(9 \widehat{\jmath} 30+)$ & $36,38,51,58,88$ \\
\hline
\end{tabular}

$18,24,26,27,28,36,37,38,39,40,42,43,45,47,51$, $53,55,57,58,60,61,62,63,67,69,71,75,76,77$, Hycleus scabiosae (A.G. Olivier, 1811) $686(331 \widehat{\jmath}, 355+q)$ $81,82,85,88,90,91,94,99,100,102,120,121,122$, $124,130,131,133,134,136,141,144,152,153,154$, $155,159,164,165,166,167,168,170,175,176,178$, 186

\begin{tabular}{|c|c|c|}
\hline Hycleus sexmaculatus (A. G. Olivier, 1811) & $7(4 ふ ふ, 39+)$ & $55,164,184$ \\
\hline Hycleus zebraus (Marseul, 1870) & $53(26 ふ \hat{\jmath}, 27+q)$ & 5, 37, 39, 124, 127, 130, 135, 162, 172, 176 \\
\hline Mylabris (Eumylabris) calida (Pallas, 1782) & $11(6 ふ ふ, 5 q 9)$ & $31,88,89,96$ \\
\hline Mylabris (E.) cincta A. G. Olivier, 1811 & $85(42 \hat{\jmath} \widehat{\jmath}, 43++q)$ & $31,33,37,89,98,122,144,145$ \\
\hline
\end{tabular}


Table 2. Male-female specimen numbers of detected species and code of collecting localities. (Continued)
Mylabris (E.) crocata (Pallas, 1781)
$79(39 ふ \hat{\jmath}, 40$ 우 $)$
$4,24,25,49,53,88,90,91,92,126,127,130,133$
$135,139,141,142,143$

\begin{tabular}{|c|c|c|}
\hline Mylabris (E.) fabricii Sumakov, 1924 & 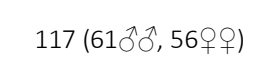 & $\begin{array}{c}24,25,31,32,33,36,37,38,39,46,48,70,78,88,89 \\
90,91,96,97,179\end{array}$ \\
\hline Mylabris (Micrabris) geminata Fabricius, 1798 & $47(21 \widehat{\jmath}$, , 26웅) & $57,101,129,140,147,152,153,154,155,162$ \\
\hline Mylabris (M.) laevicollis Marseul, 1870 & $99(41 \widehat{\jmath}, 58$, $9+1)$ & $149,150,151,163$ \\
\hline Mylabris (M.) unicolor Faldermann, 1837 & $3(2 \hat{\jmath} \hat{\jmath}, 1+1)$ & 134 \\
\hline Mylabris (s.str.) olivieri Billberg, 1813 & $135(46 ふ \hat{\jmath}, 89 q+)$ & $34,51,52,88,94,100,144,152,155,164,178$ \\
\hline Mylabris (s.str.) quadripunctata (Linnaeus, 1767) & $1129(541 \delta ึ \hat{\jmath}, 588+q+)$ & $\begin{array}{c}24,25,28,29,32,34,36,37,38,39,40,43,44,45,46, \\
48,51,53,54,57,60,63,64,68,69,77,78,83,86,88, \\
89,90,91,93,94,95,96,97,119,120,122,123,124, \\
127,128,130,133,134,135,136,137,139,141,144, \\
145,146,152,153,155,156,157,158,159,160,162, \\
163,164,167,170,171,174,176,178,183\end{array}$ \\
\hline Mylabris (s.str.) variabilis (Pallas, 1782) & $100(51 \overbrace{}^{\lambda}, 49++)$ & $34,36,37,74,86,150,162$ \\
\hline Meloe (Eurymeloe) glazunovi Pliginskij, 1910 & $1\left(1 \jmath^{\Uparrow}\right)$ & 104 \\
\hline Meloe (E.) mediterraneus J. Müller, 1925 & $2\left(2 \jmath^{\lambda}\right)$ & 1,103 \\
\hline Meloe (s.str.) proscarabaeus Linnaeus, 1758 & $1(1+9)$ & 112 \\
\hline Euzonitis quadrimaculata (Pallas, 1782) & 2 (2 우) & 37 \\
\hline Euzonitis rubida (Ménétriés, 1832) & $1(1 \precsim)$ & 122 \\
\hline Euzonitis sexmaculata (A. G. Olivier, 1789) & $7(3 \widehat{\jmath}, 4$ 웅) & $36,37,40,137$ \\
\hline Nemognatha chrysomelina (Fabricius, 1775) & $42(16 \hat{\jmath}, 26 q 0+)$ & $\begin{array}{c}24,60,62,63,65,66,72,75,79,87,164,165,175 \\
176,177,180,183,187,188,189\end{array}$ \\
\hline Stenoria (s.str.) apicalis (Latreille, 1804) & $1(1+9)$ & 84 \\
\hline Zonitis (s.str.) flava Fabricius, 1775 & $8(6 \widehat{\jmath}, 2$ 웅 & $30,39,56,148,159,163,182$ \\
\hline Zonitis (s.str.) immaculata (A. G. Olivier, 1789) & 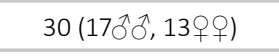 & $12,35,46,47,132$ \\
\hline Zonitis (s.str.) nana Ragusa, 1881 & $1(1+$ ) & 127 \\
\hline Stenodera (s.str.) caucasica (Pallas, 1781) & $2(29+9)$ & 107,139 \\
\hline $\begin{array}{c}\text { Stenodera (Stenoderina) anatolica (J. Frivaldszky, } \\
\text { 1884) }\end{array}$ & $1(1$ ( $)$ & 10 \\
\hline
\end{tabular}

Table 3. According to districts and geographical sections, number of species, specimen and singletons, and species diversity determined using the Brillouin diversity index ( $\mathrm{S}$ : Singleton values, these values represent the number of species with only one specimen in samples).

\begin{tabular}{|c|c|c|c|c|c|c|c|c|c|}
\hline District and Sections & $\begin{array}{l}\text { No. of } \\
\text { species }\end{array}$ & $\begin{array}{c}\text { No. of } \\
\text { specimens }\end{array}$ & S & Diversity & District and Sections & $\begin{array}{l}\text { No. of } \\
\text { species }\end{array}$ & $\begin{array}{c}\text { No. of } \\
\text { specimens }\end{array}$ & $\mathrm{S}$ & Diversity \\
\hline Akyurt & 7 & 178 & 2 & 1.241 & Gölbaşı & 10 & 115 & 4 & 1.471 \\
\hline Ayaş & 12 & 106 & 4 & 1.817 & Güdül & 3 & 69 & 0 & 0.467 \\
\hline Bala & 20 & 631 & 4 & 1.479 & Haymana & 16 & 389 & 7 & 1.781 \\
\hline Beypazarı & 9 & 84 & 2 & 1.342 & Kalecik & 8 & 55 & 2 & 1.182 \\
\hline Central & 15 & 358 & 2 & 1.897 & Kazan & 12 & 338 & 6 & 1.531 \\
\hline Çamlıdere & 1 & 1 & 1 & 0 & Kızılcaham & 8 & 123 & 3 & 0.704 \\
\hline Çubuk & 10 & 45 & 4 & 1.306 & Nallıhan & 12 & 212 & 5 & 1.559 \\
\hline Elmadağ & 3 & 22 & 1 & 0.591 & Polatlı & 18 & 343 & 5 & 1.562 \\
\hline Evren & 4 & 8 & 3 & 0.727 & Şereflikoçhisar & 13 & 253 & 4 & 1.629 \\
\hline Upper Sakarya Section & 37 & 2097 & 11 & 2.217 & Konya Section & 12 & 250 & 3 & 1.614 \\
\hline $\begin{array}{l}\text { Western Black Sea } \\
\text { Section }\end{array}$ & 12 & 280 & 3 & 1.545 & $\begin{array}{l}\text { Middle Kızılırmak } \\
\text { Section }\end{array}$ & 23 & 703 & 6 & 1.654 \\
\hline
\end{tabular}




\section{Western Black Sea Section}

37 Upper Sakarya Section

23. Middle Kızilırmak Section

12. Konya Section

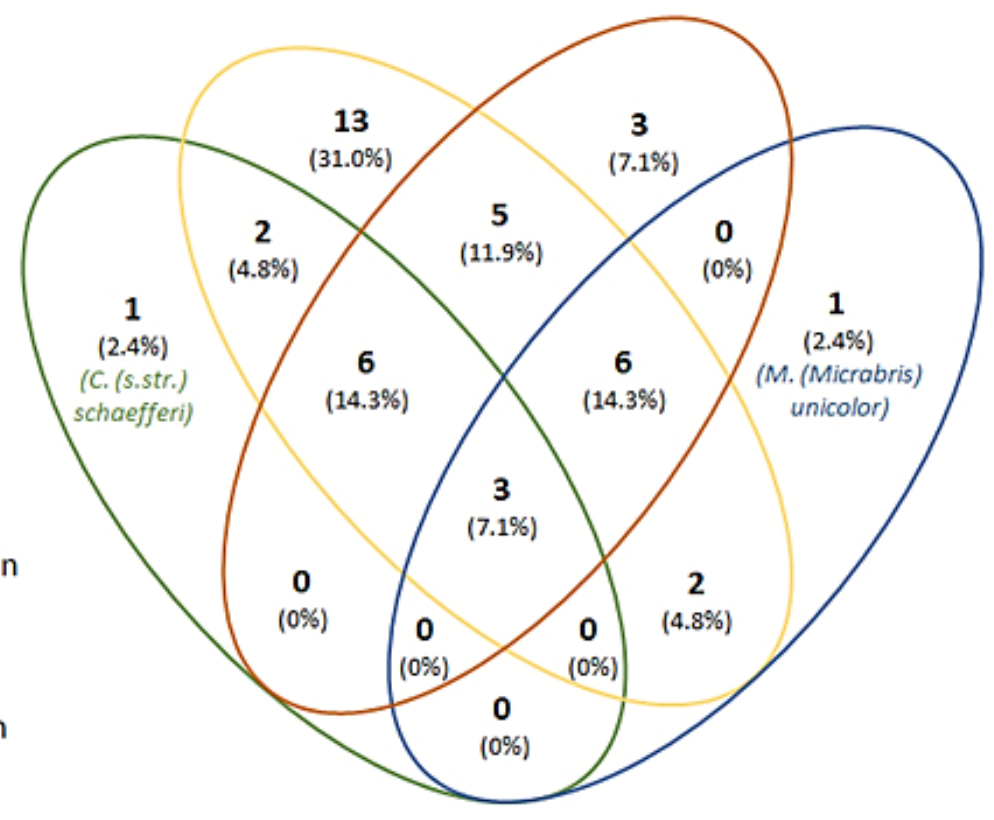

Figure 3. Turkey's geographical sections, remaining within the boundaries of Ankara, species richness of geographic sections and the number of species shared between these sections.

Table 4. Undetermined species, which are present in the current literature, in the scope of this study.

\begin{tabular}{|c|c|c|c|c|c|}
\hline & Species & Literature & & Species & Literature \\
\hline 1 & $\begin{array}{c}\text { Cerocoma (Meloides) macedonica } \\
\text { Mařan, } 1944\end{array}$ & {$[47,48]$} & 8 & $\begin{array}{c}\text { Mylabris (Mylabris) concolor Marseul, } \\
1870\end{array}$ & {$[50]$} \\
\hline 2 & $\begin{array}{l}\text { Cerocoma (M.) muehlfeldi Gyllenhal, } \\
1817\end{array}$ & {$[5,17,49]$} & 9 & Mylabris (M.) emiliae (Escherich, 1899) & [51] \\
\hline 3 & $\begin{array}{c}\text { Cerocoma (Mesocerocoma) scovitzi } \\
\text { Faldermann, } 1837\end{array}$ & {$[17,48,49]$} & 10 & $\begin{array}{c}\text { Meloe (Lasiomeloe) oliveri Chevrolat, } \\
1833\end{array}$ & {$[19,27,52-54]$} \\
\hline 4 & $\begin{array}{c}\text { Cerocoma (Metacerocoma) schreberi } \\
\text { Fabricius, } 1781\end{array}$ & [49] & 11 & Meloe (L.) hungarus Schrank, 1776 & [7] \\
\hline 5 & Lydus trimaculatus Fabricius, 1775 & [7] & 12 & $\begin{array}{c}\text { Meloe (Taphromeloe) erythrocnemus } \\
\text { Pallas, } 1782\end{array}$ & {$[7,11]$} \\
\hline 6 & Muzimes collaris (Fabricius, 1787) & [7] & 13 & $\begin{array}{l}\text { Zonitis (Zonitis) microcephala Escherich, } \\
\qquad 1897\end{array}$ & [28] \\
\hline 7 & $\begin{array}{c}\text { Mylabris (Chalcabris) munda (Escherich, } \\
\text { 1897) }\end{array}$ & [49] & 14 & Zonitis (Z.) ruficollis Frivaldsky, 1877 & [19] \\
\hline
\end{tabular}


Table 5. Undetermined species, which are present in the current literature, in the scope of this study.

\begin{tabular}{|c|c|c|c|c|c|c|c|c|c|c|c|c|c|c|}
\hline $\begin{array}{l}\text { Number of } \\
\text { species }\end{array}$ & 43 & 8 & 43 & 14 & 4 & 9 & 37 & 17 & 24 & 15 & 30 & 16 & 10 & 7 \\
\hline & 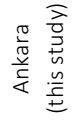 & 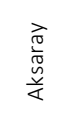 & 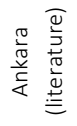 & 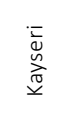 & 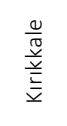 & 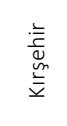 & 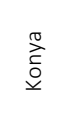 & 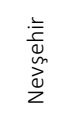 & $\begin{array}{l}\frac{0}{80} \\
\overline{200} \\
z\end{array}$ & 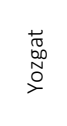 & 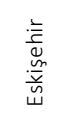 & $\stackrel{\infty}{n}_{i}^{\pi}$ & 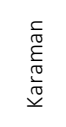 & 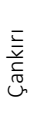 \\
\hline Ankara (this study) & 1 & & & & & & & & & & & & & \\
\hline Aksaray & 0.39 & 1 & & & & & & & & & & & & \\
\hline Ankara (literature) & 0.67 & 0.43 & 1 & & & & & & & & & & & \\
\hline Kayseri & 0.35 & 0.75 & 0.42 & 1 & & & & & & & & & & \\
\hline Kırıkkale & 0.20 & 0.49 & 0.25 & 0.61 & 1 & & & & & & & & & \\
\hline Kırşehir & 0.42 & 0.77 & 0.42 & 0.59 & 0.46 & 1 & & & & & & & & \\
\hline Konya & 0.48 & 0.44 & 0.65 & 0.53 & 0.29 & 0.43 & 1 & & & & & & & \\
\hline Nevşehir & 0.55 & 0.74 & 0.55 & 0.60 & 0.34 & 0.67 & 0.57 & 1 & & & & & & \\
\hline Niğde & 0.56 & 0.57 & 0.54 & 0.55 & 0.36 & 0.60 & 0.47 & 0.64 & 1 & & & & & \\
\hline Yozgat & 0.49 & 0.48 & 0.46 & 0.41 & 0.46 & 0.60 & 0.33 & 0.44 & 0.55 & 1 & & & & \\
\hline Eskişehir & 0.53 & 0.57 & 0.60 & 0.55 & 0.36 & 0.51 & 0.55 & 0.62 & 0.58 & 0.48 & 1 & & & \\
\hline Sivas & 0.39 & 0.62 & 0.46 & 0.60 & 0.55 & 0.60 & 0.54 & 0.60 & 0.68 & 0.50 & 0.55 & 1 & & \\
\hline Karaman & 0.34 & 0.59 & 0.41 & 0.41 & 0.44 & 0.48 & 0.46 & 0.59 & 0.49 & 0.45 & 0.41 & 0.52 & 1 & \\
\hline Çankırı & 0.32 & 0.42 & 0.32 & 0.46 & 0.52 & 0.54 & 0.41 & 0.50 & 0.40 & 0.57 & 0.39 & 0.57 & 0.51 & 1 \\
\hline
\end{tabular}

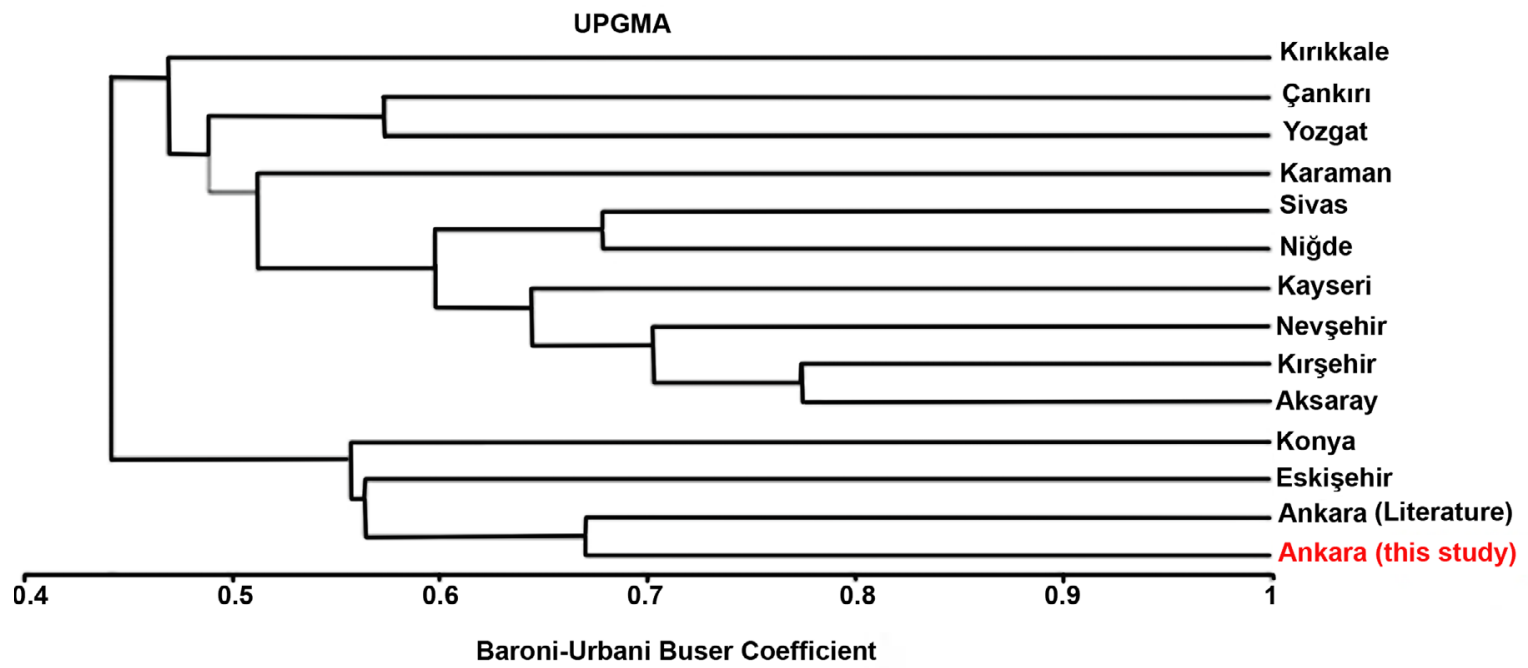

Figure 4. Dendrogram showing of the comparison between detected fauna and current literature by using the Baroni-Urbani Buser similarity index. 
ture (67\%). While it is the furthest to Meloidae fauna of Kırıkkale province (20\%).

\section{DISCUSSION}

As a result of this study, we can state that Ankara has rich Meloidae fauna with the result of this study and literature (57 species) and its fauna is $32.2 \%$ of Turkish fauna.

Evaluation of faunistic results exhibits that, ecological characteristics of Bala, Polatlı and Haymana districts such as vegetation, elevation, air temperature are supposed to be more suitable for species of Meloidae family compared to other districts. Field studies observations also support this situation. As a reason, it is thought that more homogeneous distribution may be an impressive factor in the number of identified species and samples compared to Bala, Polatlı and Haymana districts. In addition, the northern districts (eg Çamlıdere, Güdül, Kızılcahamam and Elmadağ), which are moist and rainy regions with forest vegetation, are lower in species diversity than the southern districts having steppe vegetation, represented by arid and terrestrial climate (Table 3 ).

Ankara is located at the intersection point of four geographical sections of Turkey and Meloidae faunas of these sections were examined separately. Although size of the study area and the number of locations may affect, it is thought that these sections are different in terms of their ecological characteristics such as climate, vegetation etc. affecting the species composition. Western Black Sea section high altitude, forest area and abundant rainfall; Konya Section is thought to be the driest section of Turkey and the Tuz Lake in this section has a negative effect on fauna. In addition, it is seen that the Konya and Western Black Sea sections contain only 1 special species, while the Middle Kızılırmak section contains 3 species (Figure 3 ).

According to the current literature, Meloidae fauna of Ankara province is represented by 43 species, [5, 7, 10, $11,16,17,19,28,31,32,38,47-51,53-58]$, and 43 spe cies were identified in this study, but it was observed that the species compositions were different. While $29(67.4 \%)$ of the detected species were registered in Ankara fauna in the current literature, 14 species were registered for the first time. These species are Cerocoma (s.str.) bernhaueri, Alosimus luteus, Teratolytta gentilis, T. monticola, Hycleus polymorphus, H. sexmaculatus, Mylabris (Micrabris) laevicollis Marseul, 1870, M. (Micrabris) unicolor, Meloe (Eurymeloe) glazunovi, $M$. (Eurymeloe) mediterraneus, Euzonitis rubida, Stenoria (s.str.) apicalis, Zonitis (s.str.) flava Fabricius, 1775 and Z. (s.str.) nana. As a result of the comparison of Meloidae fauna similarity between Ankara and other Central Anatolian provinces, it was found that the detected fauna overlaps with the current literature (with 67\%) (Figure 4, Table 5). In addition, it is thought that the species registered long years ago may be negatively affected by urbanization, population growth and industrialization, besides it is thought that the species may be also negatively affected by factors such as global climate change and destruction or extinction of habitats. For example, Mylabris munda and Zonitis microcephala were identified by Escherich [49] as new species from Ankara. However, these species which were endemic to Turkey weren't detected in any other study. Unfortunately, their habitats may have narrowed, disappeared or species may have been distributed to another area due to urbanization and climate change. Also, in the current literature, no information about the phenology of these species has been found. Perhaps if they are active in a very short time, it may not have been found. In this study, Lydus trimaculatus Fabricius, 1775 detected by Özbek and Szaloki [7] from Ankara could not be found, but Lydus turcicus Kaszab, 1952 was found. In the current literature $[5,36]$, it is reported that L. trimaculatus, L. quadrimaculatus Tauscher, 1812 and L. turcicus species can be confused with each other. In this study, 58 specimens from different areas of Ankara were collected and as a result of the examinations, it was determined as L. turcicus.

In addition, we added 14 species to Meloidae fauna of Ankara with this study and species numbers to 57 and $32.56 \%$ has been contributed to the number of species in the current literature. The current literature exhibited that, quite a few species are given from some provinces of Central Anatolian Region (Aksaray, Çankırı, Karaman, Kırıkkale, Kırşehir) The possible reason of this can be lack of enough comprehensive study on the Meloidae family in these provinces. Considering the results obtained from this study, it is thought that possible future studies will fill this gap in this region.

Approximately $24.3 \%$ of Turkish Meloidae fauna were detected in this study. Considering the current literature data for Ankara Meloidae fauna, it is thought that it 
would not be wrong to state that it has a rich composition in accordance with the habitat diversity within the provincial boundaries. It would be correct to adopt a habitat conservation approach, as it is not possible to protect species separately as for insects in general.

Turco and Bologna [17] stated that Cerocoma (s.str.) bernhaueri and C. (s.str.) dahli Kraatz, 1863, which is morphologically similar to each other, was very confused with each other in the past. It was also stated these species slightly overlapping in SE Turkey and NW Iran, and C. bernhaueri being a more eastern faunal element. In this study, samples were carefully examined according to taxonomic characters and diagnostic key [17], and it was determined that both species were found in the study area. This is very important in terms of information about the distributions of these species. Similarly, another species with an eastern element, Alosimus luteus, were recorded only in Southeastern Anatolian and Eastern Anatolian region of Turkey, according to current literature $[5,7,58,59]$. In this study, detection of this species from the Central Anatolian Region showed that it is most western distributed in the Palaearctic region.

Teratolytta monticola Bologna, 2006 was defined at high altitudes (1854-2400m) in Erzurum (Turkey) [39]. The name of the species is due to its adaptation to the mountain habitat [39]. With this study, the detection of this species from Ankara reveals that it is not endemic to high altitude and Erzurum, but may have a wider distribution. However, it should not be neglected that the genus Teratolytta has high sexual dimorphism, and was not examinated male specimens in this study, perhaps this sample may belong to new taxon or variation of another taxon. Since the diagnostic keys and definitions in the current literature $[15,39]$ point to Teratolytta monticola, in this study is given as this species. However, in the future, it is thought that this situation can definitely be clarified with male individuals who will gather from this area.

Additionally, Mylabris (Micrabris) unicolor and Stenoria (s.str.) apicalis detailed information about localities in Turkey are given for the first time in this study.

\section{Acknowledgments}

This study is a part of the MSc Thesis of first author and it was funded by Hacettepe University Scientific Research Projects Coordination Unit (Project No: FBA-2018-16318). We would like to thank Ali Kemal Kırçakçı and Hilal Deniz Eşer for their help in the field study. Also, we would like to thank Prof. Dr. Marco Alberto Bologna (Rome Tre University, Italy) for his literature support and help in diagnosing some species and Dr. Zhao Pan for his literature support (Hebei University-China).

\section{References}

1. M.A. Bologna, M. Oliverio, M. Pitzalis, P. Mariottini, Phylogeny and evolutionary history of the blister beetles (Coleoptera, Meloidae), Mol. Phylogenet. Evol., 48 (2008) 679-693.

2. M.A. Bologna, F. Turco, J.D. Pinto, 11.19. Meloidae Gyllenhal 1810. In Leschen RAB, Beutel RG, Lawrence JF, editors. Handbook of Zoology. Coleoptera, Beetles. Berlin, Germany, (2010) 681-693.

3. M.A. Bologna, J.D. Pinto, The Old World genera of Meloidae (Coleoptera): a key and synopsis, J. Nat. Hist., 36 (2002) 2013-2102.

4. M.A. Bologna, A. Di Giulio, Biological and morphological adaptations in the pre-imaginal phases of the beetle family Meloidae, Atti. Accad. Naz. Ital. Ent., 59 (2011) 141-152.

5. M.A. Bologna, Meloidae di Turchia. I. (Coleoptera), Fragm. Entomol., 15 (1979) 143-199.

6. C. Öncüer, Türkiye Bitki Zararlısı Böceklerinin Parazit ve Predatör Kataloğu [A catalogue of the parasites and predators of insect pests of Turkey]. Ofset Basımevi, Bornova-Izmir, 1991.

7. H. Özbek, D. Szaloki, A contribution to the knowledge of the Meloidae (Coleoptera) fauna of Turkey along with new records, Turk. J. Zool., 22(1) (1998) 23-40.

8. N. Lodos, Turkiye Entomolojisi VI (Genel, Uygulamali ve Faunistik)[Turkish Entomology VI (General, Applied and Faunistic]. E.Ü. Ziraat Fakültesi Ofset Basımevi, Bornovaİzmir, 1998.

9. M.F. Bulut, İzmir ili Meloidlerinin (Coleoptera: Meloidae) sistematiği ve faunası. MSc, Gazi University, Ankara, Turkey, 2010.

10. M.C. Korkmaz, Sündiken Dağları́nın Meloidleri (Coleoptera: Meloidae). MSc, Gazi University, Ankara, Turkey, 2015.

11. Ö. Urlu, Gazi Üniversitesi Zooloji Müzesindeki Meloidae (Coleoptera) Familyası örneklerinin değerlendirilmesi. MSc, Gazi University, Ankara, Turkey, 2006.

12. M.A. Bologna, Meloidae, in Löbl I, Smetana A, editors. Catalogue of Palaearctic Coleoptera (Volume 5: Tenebrionoidea). Apollo Books, Stenstrup, 2008.

13. M.A. Bologna, Sitarobrachys thoracica (Kraatz, 1862): new genus and species for the blister beetle fauna of Turkey (Coleoptera: Meloidae), Fragm. Entomol., 48 (2016) 125126.

14. M.A. Bologna, A. Di Giulio, Revision of the genus Trichomeloe Reitter, with the description of new species and first instar larvae (Coleoptera Meloidae), Contrib. Zool., 77 (2008) 227248.

15. M.A. Bologna, A. Di Giulio, New species of Teratolytta Semenov, 1894 from Turkey and a key to the females (Coleoptera Meloidae), ZooKeys, (625) (2016) 87-97.

16. Z. Pan, M.A. Bologna, Taxonomy, Bionomics and Faunistics of the Nominate Subgenus of Mylabris Fabricius, 1775, with the description of five new species (Coleoptera: Meloidae: Mylabrini). Zootaxa, 3806 (2014) 1-78.

17. F. Turco, M.A. Bologna, Systematic revision of the genus Cerocoma Geoffroy, 1762 (Coleoptera: Meloidae: Cerocomini), Zootaxa, 2853 (2011) 1-71. 
18. A.Ö. Koçak, M. Kemal, A new Mylabris species and subgenus for the fauna of Turkey (Coleoptera, Meloidae), Cesa News, 84 (2012) 12-14.

19. Escherich K (1896a). Meloiden-studien. IV. Theil, Wien. Entomol. Ztg., 15, 27-30.

20. Governorship of Ankara, Genel Coğrafya ve Yeryüzü Şekilleri. TC Ankara Valiliği. Available from: http://www.ankara.gov.tr/ genel-cografya-ve-yeryuzu-sekilleri (30.05.2020).

21. Governorship of Ankara, İklimi. TC Ankara Valiliği, Available from: http://www.ankara.gov.tr/iklimi (30.05.2020).

22. Turkey General Directorate of Mapping, Türkiye Harita Genel Müdürlüğü, Available from https://www.harita.gov. $\operatorname{tr} /(30.05 .2020)$.

23. Arcgis Pro v.2.2. ESRI (Environmental Systems Research Institute's), License Server: arcgis.hacettepe.edu.tr.

24. S.A.de. Marseul, Monographie des mylabrides d'Europe, et des contrées limitrophes en Afrique et en Asie, L'Abeille, Mem. d’Entomol., 7 (1870) 1-204.

25. S.A.de. Marseul, Monographie des mylabrides, Mem. Soc. R. Sci. Liege., 2(3) (1872) 363-662.

26. E. Reitter, Übersicht der Arten der Gattung Cerocoma, Deut. Entomol. Z., 29 (1885) 12-14.

27. K. Escherich, Revision der Meloïden-gattung Lydus Latr Dtsch. Entomol. Z., (1896b) 193-235.

28. K. Escherich, Revision der palaearktischen Zonitiden, einer Unterfamilie der Meloiden, Verh. Naturf. Vereins. Brünn., 35 (1897a) 96-132.

29. E. Reitter, Fauna Germanica. Die Käfer Deutschen Reiches. Nach der analytischen methode Bearb 3. Stuttgart, 1911.

30. G. Sumakov, Les espèces paléarctiques du genre Mylabris Fabr. (Coleoptera, Meloïdae), Hor. Soc. Ent. Rossicae, 42 (1915) (in French).

31. J. Mařan, Specie rum generis Lydus (Subg. Alosimus Muls.) ex affinitate speciei Lydus syriacus L. revisio. Coleoptera Meloidae, Sb. Entomol. Odd. Zem. Mus. Praze., 20 (1942) 78-98.

32. J. Mařan, Revise rodu Stenodera Eschschz. [Generis Stenodera Eschschz. revisio]. Coleoptera. Meloidae, Sb. Entomol. Odd. Zem. Mus. Praze., 20 (1942b) 14-29.

33. Z. Kaszab, Neue Revision der Gattung Alosimus Muls. (Col., Meloidae), Annls. Hist-Nat. Mus. Natn. Hung., 1 (1951) 138 150.

34. Z. Kaszab, Revision der Cerocominen, Acta Biol. Acad. Sci. Hung., 2 (1951b) 255-274.

35. Z. Kaszab, Über die Arten der Gattung Oenas, Acta Biol. Acad. Sci. Hung., 2 (1951c) 275-279.

36. Z. Kaszab, Eine neue Lydus-Art aus Kleinasien, nebst einer Bestimmungstabelle der bisher bekannten Arten der Gattung Lydus sensu stricto (Col., Meloidae), Annls. Hist-Nat. Mus. Natn. Hung., 43 (1952) 95-99.

37. S. Iablokoff-Khnzorian, Fauna Armenia SSR. Meloidae and Alleculidae. Akademia Nauk Armeniskoye SSR, Institut Zoologii, 5, 1983

38. M.A. Bologna, A. Di Giulio, J.D. Pinto, Review of the genus Stenodera with a description of the first instar larva of $S$. puncticollis (Coleoptera: Meloidae), Eur. J. Entomol., 99 (2002) 299-314.

39. M.A. Bologna, A. Di Giulio, Revision of the genus Teratolytta (Coleoptera: Meloidae), Eur. J. Entomol., 103 (2006) 137-161.

40. M.A. Bologna, A. Di Giulio, M. Pitzalis, Systematics and biogeography of the genus Actenodia (Coleoptera: Meloidae: Mylabrini), Syst. Entomol., 33 (2008) 319-360
41. Z. Pan, X. Wang, G. Ren, Taxonomy of Mylabris (Eumylabris) Kuzin (Coleoptera: Meloidae) from China. Entomotaxonomia, 32(Supplement) (2010) 34-42.

42. M.A. Bologna, Coleoptera, Meloidae, 28 Edizioni Calderini, 1991.

43. L. Brillouin, Science and information theory.Academic Press, New York, 1956.

44. Ø. Hammer, D.A. Harper, P.D. Ryan, PAST: paleontological statistics software package for education and data analysis. Palaeontologia electronica, 4 (2001) 9.

45. C. Baroni-Urbani, M.W. Buser, Similarity of binary data, Syst. Zool., 25(3) (1976) 251-259.

46. W. Kovac, MVSP - A MultiVariate Statistical Package for Windows, ver. 3.1. Kovach Computing Services, Pentraeth, Wales, U.K., 2007.

47. M. Dvořák, Taxonomische Bemerkungen zur Gattung Cerocoma Geoffroy (Coleoptera, Meloidae, Cerocomini), Annot. Zool. Bot. Bratislava, 199 (1990) 1-12.

48. J. Mařan, Vorarbeiten für eine monographische Bearbeitung der Gattung Cerocoma Geoffr. Coleoptera Meloidae, Vestn. Cesk. Spai. Zool., 9 (1944) 78-101.

49. K. Escherich, Zoologische Ergebnisse einer von Dr. K. Escherich und Dr. L. Kathariner nach Central-Kleinasien unternommenen Reise. IV. Theil. Coleopteren, Stett. Entomol. Ztg., 58 (1897) 1-69.

50. G. Sumakov, Catalogue des espěces paléarctiques de tribu Mylabrina (Coleoptera, Meloidae), Acta. Mus. Zool. Tartu., 37 (1930) 1-114 (in French).

51. K. Escherich, Zur Kenntnis der Coleopteren-Gattung Zonabris Harold, Wien. Entomol. Ztg., 18 (1899) 84-92, 97109.

52. M.A. Bologna, J.D. Pinto, The triungulin of two palaearctic Meloe subgenera: Lasiomeloe Reitter and Micromeloe Reitter (Coleoptera, Meloidae), with bionomic and taxonomic notes, Boll. Zool., 62 (1995) 383-393.

53. F. Borchmann, Meloidae, Cephaloidae "in Coleopterorum Catalogus auspeciis et auxilio S. Schenkling, pars", 1917

54. L. Ganglbauer, Coleoptera. Penther A. \& E. Zederbauer, Ergebnisse einer naturwissenschaftlichen Reise zum Erdschias-Dagh (Kleinasien), Ann. K. K. Naturhist. Hofmus., 20 (1905) 99-464.

55. Z. Kaszab, Zoologische Ergebnisse der ersten (VI.-X. 1936) und zweiten (V.-VIII. 1937) Forschungsreise N. Vasvári's in Kleinasien. IV. Meloidae, Math. Naturw. Anz. Ungar. Akad. Wiss., 60 (1941) 674-681.

56. Z. Kaszab, Wissenschafliche Ergebnisse der Zoologischen Expedition des National-Museums in Prag nach der Türkei. 25. Coleoptera: Coleoptera: Meloidae, Acta Entomol. Mus. Natl. Pragae., 33 (1959) 83-90.

57. Z. Kaszab, Ergebnisse der Albanien-Expedition 1961 des Deutschen Entomologischen Institutes. 69. Beitrag. Coleoptera: Meloidae, Beitr. Entomol., 17 (1967) 537-546.

58. S. Tezcan, Y. Karsavuran, E. Pehlivan, New Locality Records of Meloidae (Coleoptera) Fauna of Turkey, Munis Entomol. Zool., Vol. 15(No. 1) (2020) 229-234.

59. Z. Kaszab, Ergebnisse zoologischer Sammelreisen in der Türkei: Coleoptera: Meloidae, Ann. Nat. Hist. Mus. Wien., 72 (1968) 443-450 